\title{
Synthesis and investigation of the self-assembly behavior of guest block copolymers for supramolecular responsive materials
}

\author{
Shufei Jiao ${ }^{1, a}$,Yanzhen Yin ${ }^{1,2}$ b* $^{*}$, Yun Wang ${ }^{1, ~ c}$, Zhongfeng Shi ${ }^{1, d}$, Shuming \\ Zhong $^{1, e}$ and Zuqiang Huang ${ }^{2 f}$ \\ ${ }^{1}$ Guangxi Colleges and University Key Laboratory of Beibu Gulf Oil and Natural Gas Resource \\ Effective Utilization Qinzhou University, No.89, Xihuan Nanlu, Qinzhou 535000, People's Republic \\ of China. \\ ${ }^{2}$ School of Chemistry and Chemical Engineering, Guangxi University, Nanning 530004, People's \\ Republic of China. \\ ajiaoshufei2013@163.com, byinyanzhen2009@163.com, ${ }^{c}$ wangyun750@126.com, ${ }^{d}$ \\ shizhongfeng01@163.com, e zsm_7104@163.com, ${ }^{\dagger}$ huangzq@gxu.edu.cn
}

Keywords: Synthesis; Characterization; Guest copolymer; Self-assembly behavior

\begin{abstract}
A supramolecular guest copolymer (PNAP-co-PAM) based on naphthol-containing monomer (NAP) and acrylamide was synthesized via Atom Transfer Radical Polymerization. The structure of supramolecular guest copolymer was characterized by NMR and GPC. And the self-assembly behavior of supramolecular guest copolymer was revealed by optical transmittance, dynamic light scattering (DLS) and SEM. It was proved that the optimum ratio of NAP to acrylamide in PNAP-co-PAM was 8.2:1 for achieving of the nano-scaled self-assembled materials. This study provides the foundation for construction of supramolecualr responsive host-guest materials..
\end{abstract}

\section{Introduction}

Supramolecular chemistry has developed to a fascinating research field, which is used in the preparation of versatile supramolecular materials ${ }^{1,2}$. For the construction of supramolecular materials, macromolecule is one of the efficient building block ${ }^{3,4}$. Especially, versatile synthesized macromolecular building blocks was employed to construct host-guest supramolecular materials ${ }^{3,5}$. The system of cyclodextrin and adamantane (or azobenzene and naphthol) was the usual supramolecular host-guest system. Un to now, various functional host polymers based on cyclodextrin were extensively synthesized, which were applied to construct supramolecular materials based on host-guest interaction ${ }^{6,7}$. Contrastively, the synthesis of functional guest polymers was lacking $^{8}$. Typically, few functional guest polymers based on adamantane monomer were prepared and used in the development of functional materials ${ }^{8,9}$. Considering that the synthesis process of functional guest monomer was simpler than the preparing of host monomer based on cyclodextrin, the exploration of the synthesis of functional guest monomer in an efficient way is a significant goal.

Therefore, to meet such a significant challenge, the exploration of the construction of a novel guest polymer based on naphthol-containing monomer (NAP) was carried out. As far as we known, few guest polymer based on 2-naphthol monomer was reported. And just naphthol monomer was observed in the filed of chiral separations ${ }^{10}$. However, considering that naphthol was also the efficient guest molecule for the formation of complex with cyclodextrin via host-guest interaction ${ }^{11}$, the successful synthesis of guest polymers based on NAP was significant for the development of other functional supramolecular self-assembled host-guest materials. 


\section{Experiment}

\section{Materials}

2-Naphthol, 3-bromo-1-prop-anol, acrylamide, triethylamine, were purchased from J\&K scientific Ltd and were used without further purification. DMF, triethylamine, tetrahy-drofuran, ethanol were purchased from Nanning Lantian Reagent Co. Triethylamine and tetrahydrofuran were rigorously dried with sodium. Acryloyl chloride was purchased from Anhui Wotu Reagent Co. NAP was denoted by Zhejiang Yuhao Chemical Co.,Ltd. And the structure of NAP was determined as this. $\left({ }^{1} \mathrm{H}\right.$ NMR (400 MHz, $\left.\mathrm{CDCl}_{3}\right) \delta(\mathrm{ppm})$ 7.80-7.19 (7 H, naphthol), 5.87-6.17(3 H, $\left.\mathrm{CH}_{2}=\mathrm{CH}-\right)$, 4.46(t, $2 \mathrm{H}$, (naphthol, $-\mathrm{O}-\mathrm{CH}_{2}-$ ), 4.22(t, $2 \mathrm{H}, \mathrm{COOCH}_{2}-$ ), 2.27 (m, $2 \mathrm{H},-\mathrm{CH}_{2}-$ ).

\section{Instrumentation}

The characterization of the structure of the compound was performed with a Bruker $400 \mathrm{MHz}$ spectrometer using a TMS proton signal as the internal standard. UV-vis spectra were obtained using a Shimadzu 2600 UV-vis spectrophotometer. Scanning electron microscopy (SEM) observations were carried out using a JEOL JSM-6700F scanning electron microscope with a primary electron energy of $3 \mathrm{kV}$. Dynamic light scattering experiments were performed using a Malvern ZETAS12-ERNANOSERIES instrument. Molecular weights and molecular weight distributions were determined by GPC using THF as the eluent at a flow rate of $1.0 \mathrm{~mL} \cdot \mathrm{min}^{-1}$.

\section{Synthesis of supramolecular guest copolymer (PNAP-co-PAM)}

The macroinitiator PAM-Br was synthesized according to our previously report ${ }^{12}$. GPC analysis of PAM-Br revealed a Mn of 8764, Mw of 13671 and a polydispersity, Mw/Mn, of 1.56. And the degree of polymerization of PAM-Br was 119. Then, three kinds of supramolecular guest copolymer was synthesized, which were denoted as PNAP-co-PAM ${ }_{14}$, PNAP-co-PAM $_{8}$ and PNAP-co-PAM PA $_{4}$ respectively. GPC analysis of PNAP-co-PAM 14 revealed a Mn of 22711, Mw of 38679 and a polydispersity, Mw/Mn, of 1.70. And the molar ratio of acrylamide to NAP in PNAP-co-PAM 14 was 14.2:1. GPC analysis of PNAP-co-PAM $\mathbf{P}_{\mathbf{8}}$ revealed a Mn of 20342, Mw of 35502 and a polydispersity, $\mathrm{Mw} / \mathrm{Mn}$, of 1.75. And the molar ratio of acrylamide to NAP in PNAP-co-PAM $\mathbf{M}_{8}$ was 8.2:1. GPC analysis of PNAP-co-PAM 4 revealed a Mn of 21327, Mw of 37654 and a polydispersity, Mw/Mn, of 1.76. And the molar ratio of acrylamide to NAP in PNAP-co-PAM $\mathbf{P}_{4}$ was 4.1:1.

Preparation of solution of PNAP-co-PAM.

The solution of PNAP-co-PAM was prepared for the optical transmissions, DLS and SEM analysis. The concentration of PNAP-co-PAM was $0.5 \mathrm{mg} \cdot \mathrm{mL}^{-1}$.

\section{Results and Discussion}

\section{Synthesis of PNAP-co-PAM}

To explore of the preparation of functional guest monomer in an efficient way, the synthesis of a novel guest polymer based on NAP was carried out. Herein, considering that Atom Transfer Radical Polymerization (ATRP) was an efficient controlled/living radical polymerizations for the designing of versatile functional materials with complex architectures and compositions ${ }^{13}$, a series of PNAP-co-PAM were synthesized by ATRP. Typically, the synthesis route of PNAP-co-PAM was illustrated in Fig. 1. Firstly, the macroinitiator PAM-Br was synthesized using acrylamide as monomer at $30^{\circ} \mathrm{C}$. Secondly, based on the macroinitiator of PAM-Br, the block copolymer (PNAP-co-PAM) was synthesized using acrylamide and NAP as monomers at $30^{\circ} \mathrm{C}$. Through altering the molar ratio of acrylamide to NAP, a series of PNAP-co-PAM (PNAP-Co-PAM 14 , PNAP-co-PAM 8, PNAP-co-PAM 4 ) were obtained. Herein, the molar ratio of acrylamide to NAP in PNAP-co-PAM $_{14}$, PNAP-Co-PAM $_{8}$, PNAP-co-PAM $_{4}$ were 14.2:1, 8.2:1 and 4.1:1, respectively. 
<smiles>C=CC(N)=O</smiles>

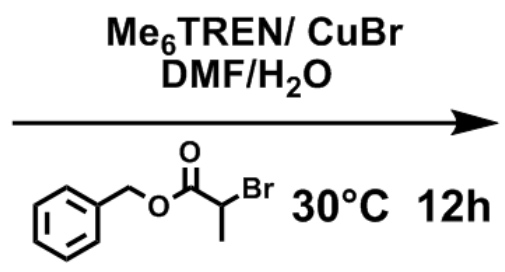<smiles>CC(CC(C)C(=O)OCc1ccccc1)C(N)=O</smiles>

\section{PAM-Br}
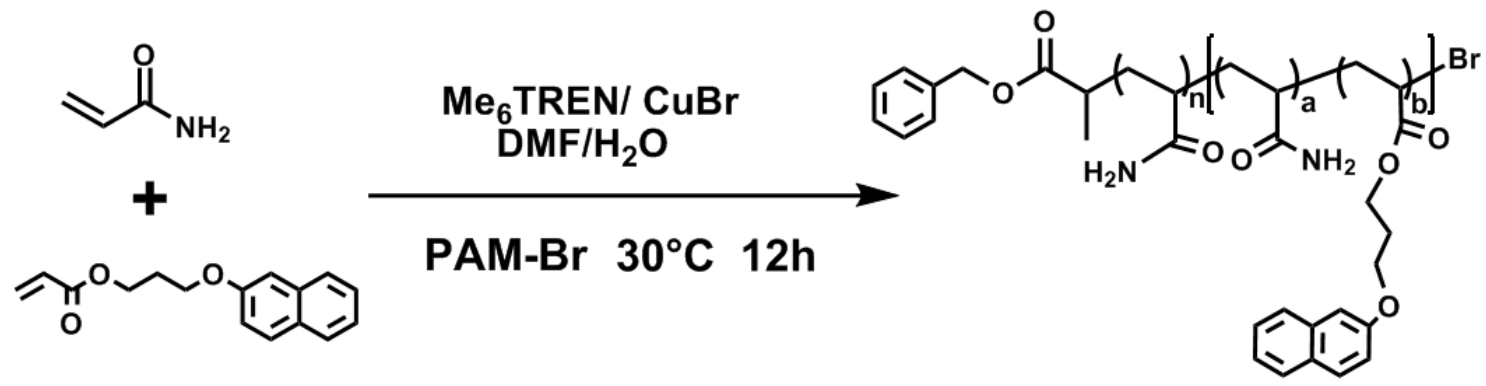

Fig. 1. The representation of the synthesis route of PNAP-co-PAM.

\section{Characterization of the self-assembly behavior of PNAP-co-PAM}

As a supramolecular guest copolymer, the reveal of the self-assembly behavior of PNAP-co-PAM is significant for the further application in various functional self-assembled materials. Herein, optical transmittance, dynamic light scattering (DLS) and SEM were employed to investigate the the self-assembly behavior of PNAP-co-PAM.

As shown in Fig. 2. the optical transmittance of the solutions of PNAP-co-PAM $\mathbf{1 4}_{14}$, PNAP-co-PAM 8 , PNAP-co-PAM ${ }_{4}$ at $600 \mathrm{~nm}$ were $67.32 \%, 8.97 \%$ and $1.82 \%$, respectively. And the hydrodynamic diameters of PNAP-co-PAM ${ }_{14}$, PNAP-co-PAM $_{\mathbf{8}}$, PNAP-co-PAM $_{4}$ were determined using dynamic light scattering (DLS), which were given in Fig.3. It was proved that the hydrodynamic diameters of the solutions of PNAP-co-PAM ${ }_{14}$, PNAP-co-PAM $_{8}$, PNAP-co-PAM $_{4}$ were about $18 \mathrm{~nm}, 110 \mathrm{~nm}$ and $950 \mathrm{~nm}$, respectively. Moreover, the actual morphologies of

PNAP-co-PAM ${ }_{14}$, PNAP-co-PAM 8 , PNAP-co-PAM 4 were investigated by SEM and illustrated in Fig. 4. It was suggested that the spherical nanoparticles were observed. However, the spherical nanoparticles of PNAP-co-PAM 14 were scarce observed and the size of them was smaller. And the size of the spherical nanoparticles of PNAP-co-PAM 4 were too large. Comparatively, the size of the spherical nanoparticles of PNAP-co-PAM $\mathbf{8}$ were about $100 \mathrm{~nm}$, which was suitable for the preparing of nano-scaled supramolecular materials.

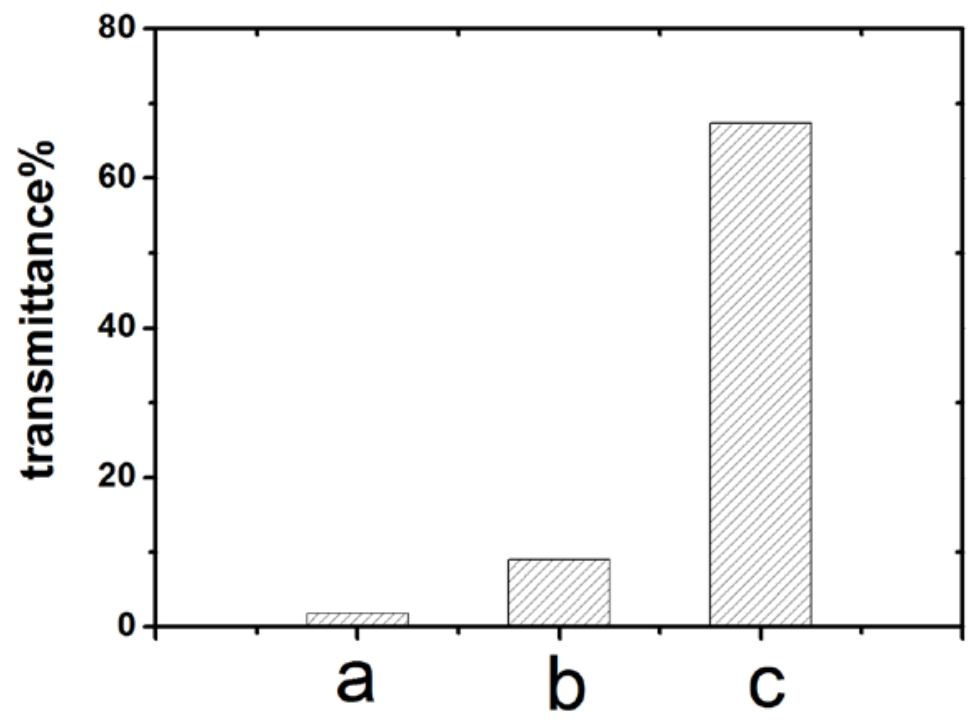

Fig. 2. The optical transmittance of three kinds of supramolecular guest copolymers at $600 \mathrm{~nm}$. (a) PNAP-co-PAM ${ }_{14}$; (b) PNAP-co-PAM 8 ; (c) PNAP-co-PAM 4. 


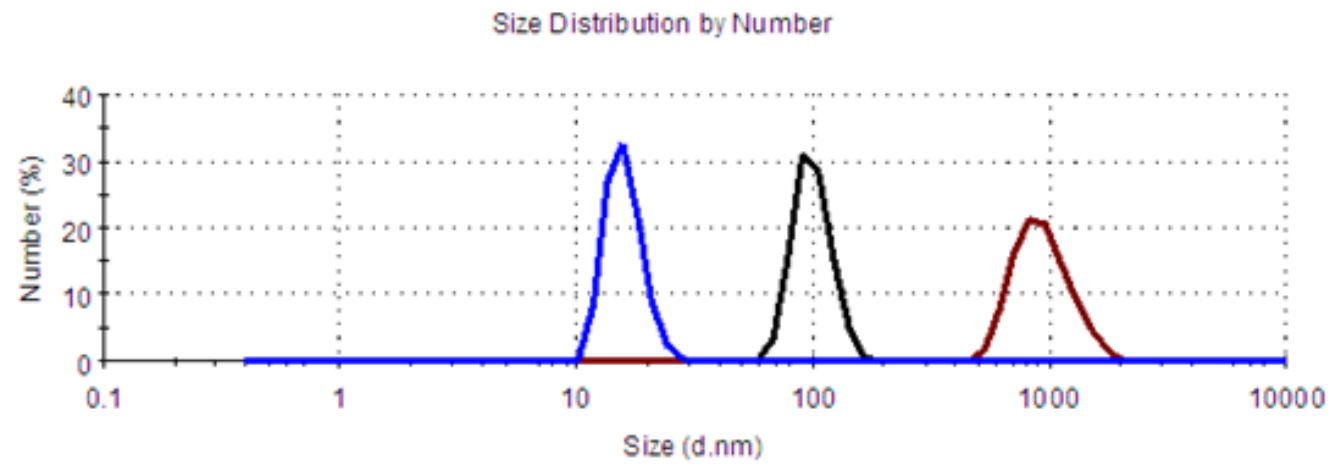

Fig. 3. Hydrodynamic diameters of three kinds of supramolecular guest copolymers determined using

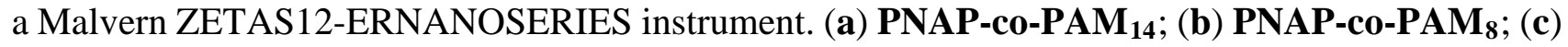
PNAP-CO-PAM . $_{\text {. }}$

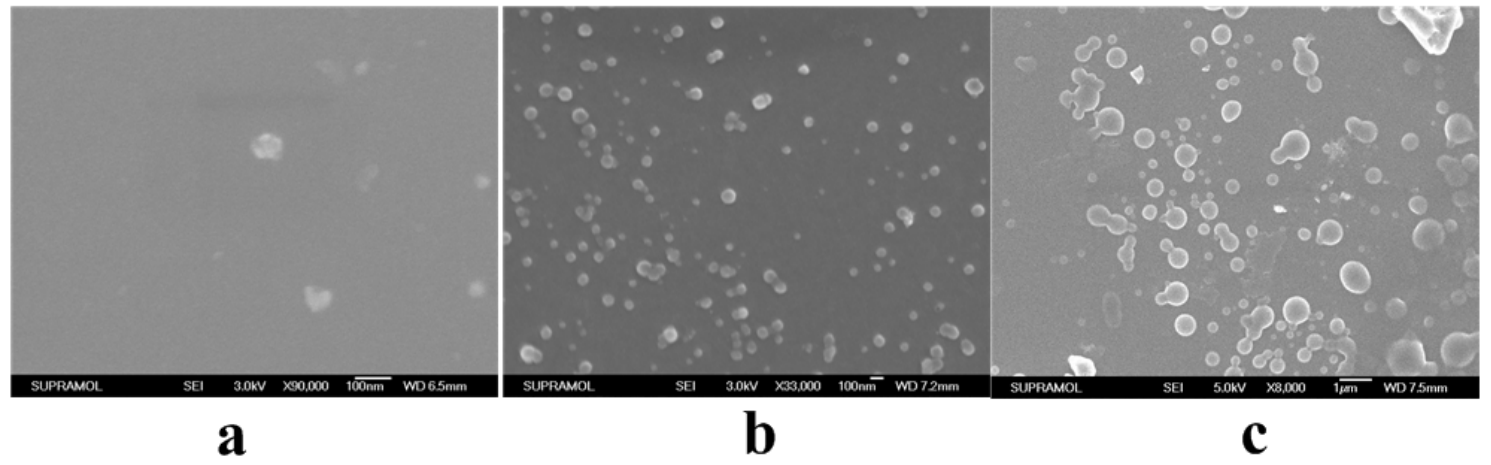

Fig. 4. SEM images of three kinds of supramolecular guest copolymers. (a) PNAP-co-PAM 14; $_{\text {; }}$ (b) PNAP-co-PAM 8 ; (c) PNAP-co-PAM 4.

\section{Discussion of the self-assembly behavior of PNAP-co-PAM}

As NAP was a hydrophobic monomer, PNAP-co-PAM (bearing NAP as functional monomer) would exhibited the enhanced hydrophobic property. Therefore, the self-assembled aggregates would be formed when PNAP-co-PAM was presented in water solution. Considering that the mole contents of NAP and the hydrophobic properties in various PNAP-co-PAM (PNAP-co-PAM 14, PNAP-co-PAM $\mathbf{8}_{\mathbf{8}}, \mathbf{P N A P - C o - P A M _ { 4 }}$ ) were different, the different aggregate structure might be observed. Herein, for PNAP-co-PAM ${ }_{14}$, the mole content of NAP was relatively low. Therefore, the optical transmittance of PNAP-co-PAM $\mathbf{1 4}_{\mathbf{1 4}}$ at $600 \mathrm{~nm}$ was $1.82 \%$, the hydrodynamic diameters was about $18 \mathrm{~nm}$, and few aggregate was observed by Fig. 4 a. It meant that the content of NAP was too low to form the efficient aggregate. Contrastly, much more large-scaled aggregates were observed when the molar ratio of acrylamide to NAP, was 4.1:1. Under this condition, the optical transmittance of PNAP-co-PAM 4 at $600 \mathrm{~nm}$ was $67.32 \%$, the hydrodynamic diameters was about $950 \mathrm{~nm}$. The self-assembled aggregate of PNAP-co-PAM 4 was not suitable for preparing of nano-scaled materials. Significantly, for PNAP-co-PAM $\mathbf{M}_{\mathbf{8}}$, the optical transmittance of PNAP-co-PAM 8 was 8.97\%, the hydrodynamic diameters was about $110 \mathrm{~nm}$, and uniform aggregates were observed by Fig. $4 \mathbf{b}$. The self-assembled aggregates of PNAP-co-PAM 8 were suitable for constructing of nano-scaled materials as a guest polymer. The successful synthesis of PNAP-co-PAM $\mathbf{8}$ was significant for the development of other functional supramolecular self-assembled host-guest materials.

\section{Conclusions}

To explore of the synthesis of functional guest monomer in an efficient way, a novel guest polymer, PNAP-co-PAM, based on naphthol-containing monomer (NAP) was synthesized. Based on the characterization of various PNAP-co-PAM (PNAP-co-PAM 14 $_{\text {, PNAP-co-PAM }}$, PNAP-co-PAM 4 ), PNAP-co-PAM 8 was selected as the suitable guest polymer for constructing of nano-scaled materials. The optical transmittance of PNAP-co-PAM $\mathbf{8}$ was $8.97 \%$, the hydrodynamic diameters was about $110 \mathrm{~nm}$, and uniform aggregates were observed by SEM. The successful 
synthesis of PNAP-co-PAM $\mathbf{8}$ was significant for the development of other functional supramolecular self-assembled host-guest materials.

\section{Acknowledgements}

This research was supported by the Natural Science Foundation of China (No: 51303088), China Postdoctoral Science Foundation (2015M582482), the project of outstanding young teachers' training in higher education institutions of Guangxi (GXQG022014063), Guangxi Distinguished Experts Special Foundation and Opening Project of Guangxi Colleges and Universities Key Laboratory of Beibu Gulf oil and Natural Gas Resource Effective Utilization(2015KLOG05, 2015KLOG14).

\section{References}

[1] G. U. Wulff and J. Liu, Accounts. Chem. Res, 45(2011) 239-247.

[2] R. Hoogenboom and U. S. Schubert, Chem.Soc. Rev, 35 (2006) 622-629.

[3] S. Yu, X. Huang, L. Miao, J. Zhu, Y. Yin, Q. Luo, J. Xu, J. Shen and J. Liu, Bioorg. Chem, 38(2010)159-164.

[4] F. van de Manakker, L. M. J. Kroon-Batenburg, T. Vermonden, C. F. van Nostrum and W. E. Hennink, Soft Matter, 6(2010)187-194.

[5] T. Ogoshi, Y. Takashima, H. Yamaguchi and A. Harada, J. Am. Chem. Soc., 129(2007)4878-4879.

[6] A. M. O’Mahony, B. M. D. C. Godinho, J. Ogier, M. Devocelle, R. Darcy, J. F. Cryan and C. M. O’Driscoll, ACS Chemi. Neurosci, 3(2012)744-752.

[7] B.K. Sarma and G. Mugesh, J. Am. Chem. Soc., 127 (2005)11477-11485.

[8] E. Blomberg, A. Kumpulainen, C. David and C. Amiel, Langmuir, 20(2004)10449-10454.

[9] C. Shen and Z. Wang, J. Phys. Chem. C, 118(1996)17585-17593.

[10] B. C. Valle, F. H. Billiot, S. A. Shamsi, X. Zhu, A. M. Powe and I. M. Warner, Electrophoresis, 25(2004)743-752.

[11] Jan van Stam, Steven De Feyter, Frans C. De Schryver and C. H. Evans, J. Phys. Chem, 100(1996)19959-19966.

[12] Y. Z. Yin, S. F. Jiao, C. Lang and J. Q. Liu, RSC Advances, 4(2014)25040-25050.

[13] J. Wang and K. Matyjaszewski, J. Am. Chem. Soc.,117(1995)5614-5615. 